PROCEEDINGS OF THE

AMERICAN MATHEMATICAL SOCIETY

Volume 131, Number 2, Pages 549-553

S 0002-9939(02)06702-3

Article electronically published on June 27, 2002

\title{
ON THE ALBANESE MAPS OF COMPACT KÄHLER MANIFOLDS
}

\author{
F. CAMPANA, T. PETERNELL, AND QI ZHANG
}

(Communicated by Michael Stillman)

\begin{abstract}
We show that compact Kähler manifolds of dimension up to four have a surjective connected Albanese map.
\end{abstract}

In this short paper, we shall study the Albanese maps on compact Kähler manifolds with numerically effective (nef) anticanonical bundles. In particular, we shall investigate the surjectivity of the Albanese maps on those manifolds.

In [DPS93, Demailly, Peternell and Schneider made the following conjecture:

Conjecture. Let $X$ be a compact Kähler manifold with $-K_{X}$ nef. Then the Albanese map $\alpha: X \rightarrow A(X)$ is surjective.

When $X$ is a projective threefold - this was proved in [DPS93] and the arbitrary higher dimensional projective cases were settled in [Zh96] by using relative deformation theory and mod $p$ reduction methods. However, in the cases of compact Kähler manifolds, a new level of sophistication is involved and things become much more difficult. In this paper, our aim is to prove the above conjecture when $X$ is of dimension three or four.

Theorem 1. Let $X$ be a compact Kähler manifold of dimension three or four such that $-K_{X}$ is nef. Then the Albanese map $f: X \rightarrow A(X)$ is surjective and has connected fibers.

The proof of Theorem 1 also provides us with the following result:

Theorem 2. Let $X$ be a compact Kähler manifold such that $-K_{X}$ is nef. Then there is no surjective map $f: X \rightarrow Y$ to a normal projective variety $Y$ of general type with $\operatorname{dim} Y \geq \operatorname{dim} X-2$.

Actually the conjecture should be true in a much stronger form:

Conjecture. Let $X$ be a compact Kähler manifold with $-K_{X}$ nef. Then $\kappa(\operatorname{det}(F))$ $\geq 0$ for all coherent subsheaves $F \subset\left(\Omega^{1} X\right)^{\otimes m}$ and all positive integers $m$.

In particular $X$ should not admit surjective maps onto normal projective varieties of positive Kodaira dimension.

Proof of Theorem 1. Let $Y=A(X)$. We may assume that $f$ has connected fibers. It is known that $X$ does not map to a curve of genus 2 or more DPS93. Thus, we may assume that $\operatorname{dim} Y>1$.

Received by the editors June 1, 1999 and, in revised form, September 27, 2001.

2000 Mathematics Subject Classification. Primary 32J27, 14K30, 14E30, 14J35.

(C)2002 American Mathematical Society 
Moreover, since every subvariety of a complex torus is an analytic fibre bundle over some other variety which is of general type, we may also assume that $Y$ is of general type Ue75.

(1) $\operatorname{dim} Y=\operatorname{dim} X$. Then $f$ is bimeromorphic, hence $\kappa(X)=\kappa(Y)$, which is absurd.

(2) $\operatorname{dim} Y=\operatorname{dim} X-1$. Let $F$ be the general fiber of $f$. We distinguish two cases according to whether $F$ is rational or elliptic (notice that $-K_{F}$ is nef).

(2.1) Suppose $F$ is rational. This case can be ruled out by [DPS93]; alternatively notice that $X$ must be projective and apply [h96].

(2.2) Suppose $F$ is elliptic. Then let $C \subset Y$ be a curve obtained as a complete intersection of general hyperplane sections of $Y$ and consider the elliptic surface $Z=f^{-1}(C) \rightarrow C$. Let $L$ denote the nef line bundle $-K_{X} \mid Z$. The key point is that $f_{*}\left(K_{Z \mid C}\right)$ is a nef line bundle and can be written in the form

$$
f_{*}\left(K_{Z \mid C}\right)=f_{*}\left(L^{*}\right)-K_{Y} \mid C
$$

where $K_{Z \mid C}$ denotes the relative canonical sheaf of $Z$ over $C$. Since $K_{Y} \mid C$ is ample, the line bundle $f_{*}\left(L^{*}\right)$ is ample. By virtue of the injective map

$$
f^{*} f_{*}\left(L^{*}\right) \longrightarrow L^{*},
$$

$L^{*}$ contains a line bundle which is semiample and neither trivial nor a torsion element. This clearly contradicts the nefness of $L$.

(3) $\operatorname{dim} Y=\operatorname{dim} X-2$. So the general fiber is now a surface with $-K_{F}$ nef, in particular $\kappa(F) \leq 0$. Again let $C \subset Y$ be a sufficiently general complete intersection of hyperplane sections and let $Z=f^{-1}(C)$.

(3.1) Suppose $\kappa(F)=0$. Then $K_{F} \equiv 0$. If $K_{F}=\mathcal{O}_{F}$, we argue exactly as in (2.2). Otherwise we find some $m \geq 2$ such that $m K_{F}=\mathcal{O}$. Now $f_{*}\left(m K_{Z \mid C}\right)$ is nef, see e.g. Ue87 and we argue by using $L=m K_{X} \mid Z$.

(3.2) If $F$ is rational, then $X$ is projective [Ca81] and the claim follows from Zh96.

(3.3) The difficult case is when $\kappa(F)=-\infty$, but $F$ is irrational. In that case $F$ is a ruled surface over an elliptic curve (given by the projectivization of a semi-stable rank 2 bundle). By Theorem 3 below, $f$ is a submersion, possibly after a base change. Moreover there is a $\mathbb{P}_{1}$ bundle structure $g: Z \rightarrow W$ and an elliptic fiber bundle structure $h: W \rightarrow C$ such that $f=h \circ g$. We are going to compute $-K_{W \mid C}$. First note - just for easier calculations - that we may assume (possibly after a finite étale base change $\tilde{W} \longrightarrow W$ ) that

$$
Z=\mathbb{P}(V)
$$

with a rank 2 vector bundle $V$ on $W$. Since $h: W \longrightarrow C$ is an elliptic submersion, we have $K_{W \mid C} \equiv 0$. Therefore

$$
K_{Z \mid C} \equiv K_{Z \mid W}
$$

and consequently we have

$$
-K_{Z \mid W}=L \otimes f^{*}\left(K_{C}\right)
$$

with a nef line bundle $L$ on $Z$. Now

$$
-K_{Z \mid W}=\mathcal{O}_{\mathbb{P}(V)}(2) \otimes g^{*} \operatorname{det} V^{*},
$$


so that $g_{*}\left(-K_{Z \mid W}\right)=S^{2} V \otimes \operatorname{det} V^{*}$. The vector bundle $S^{2} V \otimes \operatorname{det} V^{*}$ is a nef, since its tautological bundle $-K_{Z \mid W}$ is nef. Moreover $c_{1}\left(S^{2} V \otimes \operatorname{det} V^{\star}\right)=0$, the bundle $S^{2} V \otimes \operatorname{det} V^{*}$ is numerically flat [DPS93, i.e. its dual is nef, too. On the other hand, $g_{*}(L)$ is nef due to the nefness of $L$ (write $L=\mathcal{O}_{\mathbb{P}(V)}(2) \otimes g^{*}\left(L^{\prime}\right)$ ) and we have

$$
g_{*}\left(-K_{Z \mid W}\right)=g_{*}(L) \otimes h^{*}\left(K_{C}\right) .
$$

Since $g(C) \geq 2$, it is clear that $g_{*}(L)^{*} \otimes h^{*}\left(-K_{C}\right)$ cannot be nef, a contradiction. This finishes the proof of Theorem 1 .

Theorem 3. Let $Z$ be a compact Kähler threefold and $f: Z \longrightarrow C$ be a surjective map with connected fibers to a smooth curve of genus $g \geq 1$. Assume that the general fiber $F$ has $\kappa(F)=-\infty$ and $q(F)=1$. If $-K_{Z \mid C}$ is nef, then the only singular fibers of $f$ are multiples of smooth surfaces. In particular, $f$ is smooth after a finite base change $\tilde{C} \longrightarrow C$. If $C$ is elliptic, the original $f$ is already smooth. Furthermore there is a smooth minimal surface $Y, a \mathbb{P}_{1}$-bundle structure $g: Z \longrightarrow Y$ and an elliptic fibration $h: Y \longrightarrow C$ with at most multiple fibers as singular fibers such that $f=h \circ g$.

Proof. Since $-K_{F}$ is nef, $F$ is a ruled surface over an elliptic curve coming from a semi-stable vector bundle of rank 2 (this last fact will not play any role).

(1) We show that $f: Z \longrightarrow C$ admits a relative contraction $\varphi: Z \longrightarrow W$. By definition, a relative extremal contraction is a map $\varphi$ such that $-K_{Z \mid C}$ is $\varphi-$ ample, $W$ is a normal (Kähler) variety, the relative Picard number drops by 1 and there is a map $g: W \rightarrow C$ such that $f=g \circ \varphi$. In fact, first note that $f$ is locally projective, since $f$ is locally Moishezon and $Z$ is Kähler [CP98]. Furthermore $K_{Z \mid C}$ is not $f$-nef. Therefore every point $y \in C$ has an open Stein neighborhood $U$ such that a relative extremal contraction $\varphi_{U}: Z_{U} \rightarrow W_{U}$ to some normal complex space exists [Ka88]. Fix some $U$. If $\operatorname{dim} W_{U}=3$, then its center $E_{U}$-a prime divisor-must be compact, hence contained in a fiber of $f$, since the general fiber $F$ of $f: Z \rightarrow C$ is minimal. Thus we can extend $\varphi_{U}$ to a global relative contraction $\varphi: Z \rightarrow W$ by setting $\varphi=$ id outside $Z_{U}$ and gluing. So suppose that $\operatorname{dim} W_{U} \leq 2$ for all $U$. Since the general fiber of $\varphi_{U}$ has to be rational, we have $\operatorname{dim} W_{U}=2$ for all $U$. Moreover $\varphi_{U} \mid F$ is the unique $\mathbb{P}_{1}$-bundle structure on $F$. Therefore all $\varphi_{U}$ glue to a relative contraction $\varphi: Z \rightarrow W$ with $\operatorname{dim} W=2$. This proves the existence of $\varphi$.

(2) Repeating step (1) if $\varphi$ is bimeromorphic (note that $X$ is uniruled), we obtain a sequence

$$
\varphi_{i}: Z_{i} \longrightarrow Z_{i+1}
$$

where we set $Z_{0}=Z, Z_{1}=W, \varphi_{0}=\varphi$ and where every $\varphi_{i}$ is either a relative contraction or a flip (note that flips are constructed locally analytically, so there is no problem with the Kähler situation). We obtain some $r$ such that $\operatorname{dim} Z_{r+1} \leq 2$. Since the general fiber $F$ of $f$ is not touched by all these contractions and flips (note: $C$ is irrational and $F$ does not contain (-1)-curves!) and since $q(F)=1$, we conclude that $\operatorname{dim} Z_{r+1}=2$. We set, partly just for simplicity

$$
Y=Z_{r+1}, X=Z_{r}, \psi=\varphi_{r}
$$

and let $\alpha: Y \longrightarrow C$ denote the induced map.

(3) As in PS97, we say that a line bundle $L$ on a space $Z$ is almost nef if $L \cdot C \geq 0$ for all curves $C \subset Z$ with finitely many exceptions, all being rational. Since all 
contractions take place in fibers over $C$, the arguments of [PS97, 2.1, 2.2] apply and we conclude that $-K_{X \mid C}$ is almost nef. We examine the structure of $\psi$. First notice that generically, i.e. outside the singularities of $Y, \psi$ is a conic bundle. Let $\Delta$ denote the closure of the discriminant locus. Then we prove that

$$
-\left(4 K_{Y \mid C}+\Delta\right)
$$

is almost nef. Notice that $W$ is $\mathbf{Q}$-Gorenstein with only rational singularities KMM85 (see also KoMiMo92). So the above claim really makes sense. Now,

$$
\begin{aligned}
\psi_{*}\left(K_{X \mid C}^{2}\right) & =\psi_{*}\left(\left(K_{X}-\psi^{*} \alpha^{*}\left(K_{C}\right)\right)^{2}\right)=\psi_{*}\left(K_{X}^{2}-4(g-1) F\right) \\
& =\psi_{*}\left(K_{X}\right)^{2}+8(g-1) \alpha^{-1}(c)
\end{aligned}
$$

for some $c \in C$. Arguing as in PS97, 1.16], $\psi_{*}\left(K_{X}\right)^{2}=-\left(4 K_{Y}+\Delta\right)$, hence we conclude

$$
\psi_{*}\left(K_{X \mid C}^{2}\right)=-\left(4 K_{Y \mid C}+\Delta\right),
$$

hence, again as in [PS97, 1.16], $-\left(4 K_{Y \mid C}\right)+\Delta$ is almost nef.

(4) From (3) we deduce that $-4\left(K_{Y \mid C}+\Delta\right)$ is the sum of an effective and an almost nef $\mathbf{Q}$-Cartier Weil divisor. Let

$$
\pi: \hat{Y} \longrightarrow Y
$$

be the minimal desingularisation. So

$$
-K_{\hat{Y} \mid C}=\pi^{*}\left(-K_{Y \mid C}\right)+A
$$

with an effective divisor $A$ (possibly 0 ). Hence $-K_{\hat{Y} \mid C}$ is again the sum of an effective and an almost nef divisor. Let $\beta: \hat{Y} \longrightarrow C$ be the induced projection map. Then $\beta_{*}\left(K_{\hat{Y} \mid C}\right)$ is a nef line bundle, moreover

$$
\beta^{*} \beta_{*}\left(K_{\hat{Y} \mid C}\right) \subset K_{\hat{Y} \mid C} .
$$

So $K_{\hat{Y} \mid C}$ is the sum of an effective and a nef divisor, too. We conclude immediately that $K_{\hat{Y} \mid C} \equiv 0$, hence that $K_{Y \mid C} \equiv 0$; moreover $\Delta=0$ and $A=0$. Now $\beta_{*}\left(K_{\hat{Y} \mid C}\right) \equiv 0$, hence $\beta$ has only multiple fibers as singular fibers (see e.g. [BPV84]). In particular $\hat{Y}=Y$, so that $Y$ is smooth and minimal and $\alpha$ has at most multiple singular fibers. Since $\Delta=0, \psi$ is analytically a $\mathbb{P}_{1}$-bundle.

(5) We show that $\varphi_{r-1}$ cannot exist without destroying the property that $-K_{Z_{r-1 \mid C}}$ is almost nef. In case $\varphi_{r-1}$ contracts a divisor to a point $p$, we argue as follows. It is clear that $-K_{Z_{r-1} \mid C}$ is not nef (compute the top self-intersection number). Let $F$ be the fiber of $X \longrightarrow C$ containing $p$ and denote $\hat{F}$ its strict transform in $Z_{r-1}$. The map $\hat{F} \longrightarrow F$ is the blow-up of some ideal with support $p$. Now $-K_{Z_{r-1} \mid C}$ is by assumption almost nef. On the other hand $-K_{Z_{r-1} \mid C}=\varphi_{r-1}^{*}\left(-K_{F}\right)-A$ where $A$ is a divisor supported on the exceptional locus of $\hat{F} \longrightarrow F$. Let $h: \tilde{F} \longrightarrow \hat{F}$ be a desingularisation. Since the normalisation of $\hat{F}$ clearly has at most rational singularities, we conclude that $h^{*}\left(\varphi_{r-1}^{*}\left(-K_{F}\right)-A\right)$ is almost nef. This is easily contradicted by the fact that $F$ is a ruled surface over an elliptic curve, using PS97. 1.4]. We are left with the case that $\varphi_{r-1}$ is the blow-up of a smooth curve $B \subset X$. Then [PS97, 4.11] already proves that $-K_{Z_{r-1} \mid C}$ is not nef. If $B$ is contained in a fiber $F$, then we immediately compute that $-K_{Z_{r-1}} \mid \hat{F}=\varphi_{r-1}^{*}\left(-K_{F}\right)-A$, where $\hat{F}$ is the strict transform of $F$ and $A$ is exactly supported on the exceptional divisor of $\hat{F} \longrightarrow F$. Hence $-K_{Z_{r-1} \mid C}$ is not almost nef [PS97, 1.4]. If finally $B$ maps onto 
$C$, take any general fiber $F$ and then $\hat{F}$ is the blow up of $F$ in some disjoint points and it is obvious that $-K_{Z_{r-1} \mid C}$ is not almost nef.

We therefore conclude that $Z=X$ and the proof of Theorem 3 is complete.

\section{ACKNOWLEDGMENT}

Part of this work was done during the third author's stay at the Université de Nancy and the Universität Bayreuth. He would like to thank both institutes for their hospitality and their financial support. He was also partially supported by a grant from NSA. We would also like to thank the referee for pointing out an error in an earlier version of this paper as well as for some other helpful suggestions.

\section{REFERENCES}

[BPV84] Barth, W.; Peters, C.; Van de Ven, A.: Compact complex surfaces. Erg. d. Math. 3. Folge, Band 4, Springer 1984. MR 86c:32026

[Ca81] Campana, F.: Coréduction algébrique d'un espace faiblement Kählerien compact. Inv. Math. 63, 187-223 (1981). MR 84e:32028

[CP98] Campana, F; Peternell, Th.: Complex threefolds with non-trivial holomorphic 2forms, J. Algebraic Geom. 9, 223-264 (2000). MR 2002a:32016

[DPS93] Demailly, J.P.; Peternell, Th; Schneider, M.: Kähler manifolds with numerically effective Ricci class. Comp. Math. 89, 217-240 (1993). MR 95b:32044

[Ka88] Kawamata, Y.: The crepant blow-ups of 3-dimensional canonical singularities and its application to degeneratioon of surfaces. Ann. Math. 127, 93-163 (1988). MR 89d:14023

[KoMiMo92] Kollár, J.; Miyaoka, Y.; Mori, S.: Rationally connected varieties. J. Alg. Geom. 1, 429-448 (1992). MR 93i:14014

[KMM85] Kawamata, Y.; Matsuda, K.; Matsuki, K.; Introduction to the minimal model problem. Advanced Studies in Pure Mathematics 10, Algebraic Geometry, Sendai, 283360 (1985). MR 89e:14015

[PS97] Peternell, Th.; Serrano, F.: Threefolds with nef anticanonical bundles. Collectanea Math. 49, 465-517 (1998). MR 2000b:14020

[Ue75] Ueno, K.: Classification theory of algebraic varieties and compact complex spaces. Lect. Notes in Math. 439, Springer 1975. MR 58:22062

[Ue87] Ueno, K.: On compact analytic threefolds with non-trivial Albanese tori. Math. Ann. 278, 41-70 (1987). MR 88m:32054

[Zh96] Zhang, Q.: On projective manifolds with nef anticanonical bundles. J. Reine Angew. Math. 478, 57-60 (1996). MR 97m:14039

Département de Mathématiques, Université de Nancy, 54506 Vandoeuvre les Nancy, France

Mathematisches Institut, Universität Bayreuth, 95440 Bayreuth, Germany

Department of Mathematics, University of Missouri, Columbia, Missouri 65211 